\title{
Aplicação de técnicas cromatográficas e espectrométricas como ferramentas de auxílio na identificação de componentes de óleos voláteis
}

\author{
Claudia B. Brochini' ${ }^{1}$ João Henrique G. Lago ${ }^{2 *}$ \\ ${ }^{1}$ Instituto de Química, Universidade de São Paulo, Caixa Postal 26077, \\ 05513-970, São Paulo, SP, Brasil, \\ ${ }^{2}$ Centro de Ciências e Humanidades, Universidade Presbiteriana Mackenzie, \\ 01302-907, São Paulo, SP, Brasil
}

\begin{abstract}
RESUMO: A metodologia usual aplicada para análise de óleos voláteis, tais como CG, CGEM, Índice de Kovats e, em alguns casos, sistemas computacionais especialistas, não permitem a identificação de compostos desconhecidos os quais devem ser isolados para que tenham suas estruturas moleculares elucidadas. Essa limitação é ainda maior quando tais óleos apresentam potencial biológico uma vez que existe grande dificuldade no isolamento das substâncias ativas, as quais devem ser obtidas em seu estado puro para avaliação da atividade. Assim, este trabalho descreve uma metodologia de separação não usual (cromatografia rápida em sílica e sílica impregnada com $\mathrm{AgNO}_{3}$ ) associada a técnicas espectrométricas (EM, RMN de ${ }^{1} \mathrm{H}$ e RMN de ${ }^{13} \mathrm{C}$ ) as quais permitiram a purificação e caracterização de compostos de óleos voláteis contendo estruturas ainda não conhecidas e/ou biologicamente ativas.
\end{abstract}

Unitermos: Óleo volátil, separação cromatográfica, análise espectrométrica.

\begin{abstract}
Use of chromatographic and spectrometric techniques to help the identification of volatile compounds". The usual techniques applied for analyzing the chemical constitution of volatile oils, such as GC, GC-MS, Kovats Index and, in some cases, specialist computational systems, do not allow the identification of the unknown or biologically active compounds which must be isolated in order to have their molecular structures elucidated. This problem is higher if the oils present biological potential, which will induce in the isolation of active substances, once these substances must be obtained in their pure state to activity evaluation. Thus, this paper describes an unusual chromatographic separation (flash chromatography on $\mathrm{SiO}_{2}$ and $\mathrm{SiO}_{2} / \mathrm{AgNO}_{3}$ ) associated to spectrometric techniques (MS, ${ }^{1} \mathrm{H}$ NMR and ${ }^{13} \mathrm{C}$ NMR), which lead to the purification and structural elucidation of the unpublished and/or biologically active constituents from volatile oils.
\end{abstract}

Keywords: Volatile oil, chromatographic separation, spectrometric analysis.

\section{INTRODUÇÃO}

A maioria dos artigos publicados visando à identificação de componentes de óleos voláteis descreve o uso de CG e de CG/EM em associação com a determinação do índice de Kovats (Tavares et al., 2005; Mesquita et al., 2005; Oliveira et al., 2005). Este procedimento vem sendo aplicado com sucesso na identificação de substâncias de estruturas conhecidas porque, em sua maioria, os dados gerados podem ser comparados diretamente com os valores de tempo de retenção (índice de retenção) obtidos em colunas de polaridades diferentes e com os espectros de massas dos constituintes voláteis publicados (Adams, 1995). Trabalhos descritos na literatura também demonstram que sistemas especialistas computacionais são úteis na identificação de componentes de estruturas conhecidas em óleos voláteis brutos (Ferreira et al., 2001).
Não obstante, nenhum dos sistemas usados permitiu ainda a identificação dos compostos desconhecidos, os quais devem ser isolados a fim de terem suas estruturas moleculares elucidadas. Além disso, o uso dessas ferramentas é muito limitado aos grupos que as possuem ou desenvolveram uma base de dados espectroscópicos. Por outro lado, a análise dos espectros de RMN obtidos de misturas complexas, torna-se limitada para identificar todos os componentes, especialmente àqueles presentes em quantidades reduzidas. Do mesmo modo, a complexidade da composição dos óleos voláteis é um fator limitante para a utilização da técnica RMN-DOSY, usada para análise de misturas (Souza; Laverde, 2002).

Do ponto de vista farmacológico, a literatura descreve o elevado potencial biológico apresentado pelos óleos voláteis de diversas espécies vegetais, principalmente atividade antibacteriana e antifúngica 
(Costantin et al., 2001; Navickiene et al., 2006; Nascimento et al., 2007). Ao contrário do observado para estudos fitoquímicos de extratos vegetais bioativos (Lago et al., 2004a; Moreira et al., 2007), poucos são os estudos que reportam qual(is) a(s) substância(s) responsável(is) pelas atividades em óleo voláteis. Assim, torna-se fundamental conhecer a classe e/ou a identidade dos componentes ativos através de técnicas espectrométricas, somente possível após separação e purificação.

Para tanto, uma excelente alternativa é o uso da cromatografia a gás preparativa, que permite o isolamento das moléculas em sua forma pura, possibilitando a identificação indubitável de suas estruturas moleculares (König et al., 1996; Adio et al., 2002). No entanto, o custo do equipamento ainda é um fator limitante para a utilização dessa técnica como ferramenta de rotina em laboratórios de pesquisa.

Assim sendo, nosso grupo de pesquisa desenvolveu uma metodologia que se baseia em separações cromatográficas e nas análises espectrométricas para analisar misturas de sesquiterpenos isolados de óleos voláteis brutos (Brochini et al., 1999). No entanto, essa metodologia vem sendo usada com sucesso na identificação de compostos conhecidos, porém com duas limitações importantes:

1. Substâncias de estruturas desconhecidas/inéditas não puderam ter sua identidade revelada;

2. Substâncias com potencial biológico não puderam ser isoladas, mas detectadas apenas em frações enriquecidas.

Desta forma, este artigo descreve um aperfeiçoamento dessa metodologia a fim de que essas substâncias possam ser purificadas e, conseqüentemente, submetidas a técnicas espectrométricas, visando à caracterização de substâncias inéditas e/ou determinação da atividade biológica em potencial.

\section{METODOLOGIA}

Inicialmente, o óleo volátil, potencialmente ativo ou não, deve ser analisado por CG/EM seguido do cálculo do índice de Kovats em colunas cromatográficas de diferentes polaridades, conforme a metodologia convencional (Adams et al., 1995). Este procedimento deve permitir a identificação do maior número possível de constituintes. Ao se observar que determinados compostos não puderam ser identificados com segurança através desse procedimento, torna-se necessária a separação do óleo volátil bruto. Este deve ser submetido à cromatografia rápida (gel de sílica sob pressão) utilizando-se solventes voláteis tais como $\mathrm{CH}_{2} \mathrm{Cl}_{2}$ e misturas de $\mathrm{CH}_{2} \mathrm{Cl}_{2}: \mathrm{MeOH}$ nas proporções 99:1, 97:3 e 9:1. As frações similares devem ser reunidas após análise do cromatograma obtido por CG.

As frações menos voláteis (mais polares), visto que os componentes têm geralmente funções oxigenadas, podem ser submetidas aos procedimentos

cromatográficos usuais (coluna e CCDP em gel de sílica) enquanto que as mais voláteis (menos polares), constituídas geralmente por compostos não-oxigenados contendo ligações duplas, podem ser separados, de forma diferencial, através de cromatografia em coluna utilizando gel de sílica impregnada com 15\% de $\mathrm{AgNO}_{3}$ (Gupta; Dev, 1963). Neste caso, a fase móvel deve possuir baixos pontos de ebulição, tais como pentano, pentano: $\mathrm{CH}_{2} \mathrm{Cl}_{2}$ (1:1), $\mathrm{CH}_{2} \mathrm{Cl}_{2}$ puro e misturas de $\mathrm{CH}_{2} \mathrm{Cl}_{2}$ :acetona (99:1, 95:5 e 9:1). No entanto, em ambos os casos, a separação deverá ser sempre monitorada através de cromatografia a gás para que seja avaliado o grau de pureza das frações obtidas.

As análises dos espectros de RMN das frações obtidas deste procedimento confirmarão de forma inequívoca as estruturas dos componentes identificados previamente através do CG/EM e pelo índice de Kovats, podendo, inclusive, possibilitar a correção de estrutura. Deve-se ressaltar que os compostos não identificados podem corresponder a estruturas não usuais ou inéditas. Neste caso, as frações que os contém deverão ser submetidas a técnicas cromatográficas, seguida de análises espectrocópicas. De modo análogo, no caso em que o óleo for biologicamente ativo, as frações obtidas deverão ser reavaliadas quanto à atividade e, posteriormente, proceder-se-á a separação dos constituintes.

\section{RESULTADOS E DISCUSSÃO}

\section{Óleo volátil bruto contendo substância inédita}

A análise do cromatograma obtido por CG do óleo volátil bruto das folhas de Guarea macrophylla (Meliaceae) mostrou a ocorrência de 14 constituintes principais (em uma relação maior de 1\%) (Lago; Roque, 2002). Assim sendo, nas condições de trabalho, os valores de tempo de retenção sugerem a presença de hidrocarbonetos sesquiterpênicos e sesquiterpenóides bem como de diterpenos mono-oxigenados (Brochini et al., 1999; Patitucci et al., 1995). O espectro de RMN de ${ }^{1} \mathrm{H}$ do óleo bruto mostrou sinais entre $\delta_{\mathrm{H}} 0,8$ - 2,5 e entre $\delta_{\mathrm{H}} 4,8$ - 5,4, característicos de hidrogênios alifáticos, confirmando a ausência de compostos aromáticos. A análise por CG/EM bem como o cálculo do índice de Kovats do material bruto permitiu a identificação dos sesquiterpenos $\alpha$-cubebeno, $\alpha$-ylangeno, $\alpha$ copaeno, $\alpha$-gurjuneno, $\beta$-cariofileno, $\alpha$-humuleno, alo-aromadendreno, germacreno $\mathrm{D}, \gamma$-cadineno, $\delta$ cadineno, germacreno-D-4 $\alpha$-ol e ledol, correspondentes a aproximadamente 35,4 \% (em massa) do óleo volátil bruto. Uma vez que um dos derivados majoritários não foi adequadamente identificado através dessa técnica, o óleo bruto foi submetido à metodologia cromatográfica supracitada, visando o isolamento desse componente.

Assim, o material bruto foi submetido à cromatografia rápida (gel de sílica) cuja coluna foi eluida com $\mathrm{CH}_{2} \mathrm{Cl}_{2}$ puro e misturas compostas por 1 , 3 e $10 \%$ 
de $\mathrm{MeOH}$ em $\mathrm{CH}_{2} \mathrm{Cl}_{2}$. Esse procedimento forneceu 64 frações (6 mL cada), que foram combinadas em 10 grupos após análise de seus cromatogramas obtidos por CG. O grupo 1, composto por uma mistura de hidrocarbonetos sesquiterpênicos, foi submetido a uma coluna de gel de sílica impregnada com $\mathrm{AgNO}_{3}$ usando pentano, 50\% de $\mathrm{CH}_{2} \mathrm{Cl}_{2}$ em pentano e misturas de 1, 5 e $10 \%$ de acetona em $\mathrm{CH}_{2} \mathrm{Cl}_{2}$ como fases móveis. Este procedimento resultou em 49 frações, que foram analisadas por CG e então reunidas em 7 grupos. Através da análise por RMN foram identificados os sesquiterpenos alo-aromadendreno, germacreno $\mathrm{D}$ e $\beta$-cariofileno, obtidos na forma pura, além de misturas compostas por $\beta$-cariofileno + espatulenol e alo-aromadendreno + isopimara-7,15-dieno.

A análise os dados de $\mathrm{RMN}$ de ${ }^{1} \mathrm{H}$ e de ${ }^{13} \mathrm{C}$ dos grupos 2 a 7, mostrou que os mesmos eram constituídos, respectivamente, pelos diterpenos óxido de manoíla e isopimara-7,15-dien-3-ona, o qual foi isolado pela primeira vez de fonte natural, e pelos sesquiterpenos guai6-en-10 $\beta$-ol, palustrol, germacreno-D-4 $\alpha$-ol e ledol, todos obtidos de forma pura. Os sesquiterpenos guai-6-en-10 $\beta$ ol e ledol são os constituintes principais do óleo (17,3\% e $13,9 \%$, respectivamente), sendo que esse último já havia sido identificado através da análise por CG/EM do óleo volátil bruto. É importante observar que seria impossível caracterizar o sesquiterpeno guai-6-en-10 $\beta$-ol através de $\mathrm{CG} / \mathrm{EM}$ uma vez que este composto foi isolado apenas do extrato em $\mathrm{CH}_{2} \mathrm{Cl}_{2}$ das folhas de G. macrophylla (Lago et al., 2000), ou seja, sem que seus dados estejam relatados nas espectrotecas de massas disponíveis no mercado.

\section{Óleo volátil bruto apresentando potencial biológico}

A avaliação da inibição do crescimento de $S$. aureus e de $E$. coli frente aos óleos voláteis das folhas e das cascas dos galhos de Cedrella fissilis (Meliaceae) mostrou que o apenas óleo bruto obtido das folhas apresentou moderada atividade antibacteriana. Com o objetivo de se reconhecer a identidade molecular dos componentes, os óleos foram analisados por CG/EM associado ao cálculo do índice de Kovats o qual possibilitou a caracterização, com segurança, de 17 componentes no óleo das folhas e 23 componentes no óleo das cascas dos galhos (Lago et al., 2004b). Os óleos voláteis foram também analisados por RMN de ${ }^{1} \mathrm{H}$, que devido aos sinais entre $\delta_{\mathrm{H}} 0,8$ - 2,4 e entre $\delta_{H} 4,9-5,4$, indicaram natureza terpênica.

O fracionamento cromatográfico do óleo das folhas foi realizado com duplo objetivo, o de se confirmar à identificação dos componentes além de possibilitar a caracterização da(s) substância(s) ativa(s). Assim, o óleo foi fracionado em coluna rápida (gel de sílica) eluida com pentano, $\mathrm{CH}_{2} \mathrm{Cl}_{2}$ e mistura de $\mathrm{CH}_{2} \mathrm{Cl}_{2}$ : $\mathrm{MeOH}$ (99:1) sendo obtidas 46 frações (8 mL cada) as quais foram reunidas em 8 grupos após análise por CG. Desta forma, após análise por $\mathrm{RMN}$ de ${ }^{1} \mathrm{H}$ e ${ }^{13} \mathrm{C}$, foi possivel caracterizar os componentes espatulenol, vididiflorol, globulol, e $\tau$-muurolol dos grupos 4, 6, 7 e 8 , respectivamente. $\mathrm{O}$ grupo 1, após fracionamento em coluna de gel de sílica impregnada com $\mathrm{AgNO}_{3}$, a qual foi eluida com pentano, $\mathrm{CH}_{2} \mathrm{Cl}_{2}$ e $\mathrm{CH}_{2} \mathrm{Cl}_{2}: \mathrm{Me}_{2} \mathrm{CO}$ (99:1), forneceu 34 frações (7 $\mathrm{mL}$ cada) que foram reunidas em 7 grupos após análise por CG. Assim, após análise por RMN, os grupos 3, 4 e 6 mostraram-se constituídos por biciclogermacreno, $\beta$-cariofileno e $\beta$-bisaboleno, respectivamente. Tal procedimento possibilitou a confirmação da identidade molecular desses componentes, os quais haviam sido anteriormente identificados por CG-EM associado ao cálculo do índice de Kovats.

Finalmente, para se verificar quais os componentes responsáveis pela atividade antibacteriana observada, todos os grupos (1 - 8) obtidos da coluna de $\mathrm{SiO}_{2}$ foram avaliados quanto à inibição do crescimento dos microorganismos testados, sendo verificado que o grupo 1 mostrou maior potencial. Uma vez que este foi submetido a novo processo de separação, as 7 frações provenientes da coluna de gel de sílica impregnada com $\mathrm{AgNO}_{3}$ foram também avaliadas no bioensaio em questão. Destas, aquelas que apresentaram maior potencial foram as 3 e 4 , compostas, respectivamente, por biciclogermacreno e $\beta$-cariofileno.

\section{Óleo volátil bruto contendo substância inédita e potencial biológico}

O óleo volátil bruto das folhas de Guarea guidonia (Meliaceae) apresentou moderada atividade antifúngica frente à Cladosporium cladosporioides e C. sphaerospermum (Homas; Fuchs, 1970; Rahalison et al., 1994). O cromatograma do óleo bruto, obtido através de CG, mostrou a presença de nove constituintes em uma proporção maior que 1\%. A análise por CG/EM associada ao cálculo do índice de Kovats possibilitou a identificação dos sesquiterpenos $\delta$-elemeno, $\alpha$-guaieno, eudesma-4,11dieno, $\beta$-selineno e eudesm-6-en-4 $\beta$-ol, correspondendo a 36\% do óleo volátil bruto (Lago et al., 2002). Os valores de tempo de retenção observados sugeriram a presença de hidrocarbonetos sesquiterpênicos e sesquiterpenos mono e di-oxigenados (Brochini et al., 1999; Patitucci et al., 1995). A cromatografia rápida (gel de sílica) do material bruto, eluida com $\mathrm{CH}_{2} \mathrm{Cl}_{2}$ forneceu 55 frações $(6 \mathrm{~mL}$ cada), que foram combinadas em 10 grupos após a análise individual dos cromatogramas obtidos por CG. A análise dos espectros de $\mathrm{RMN}$ de ${ }^{1} \mathrm{He}^{13} \mathrm{C}$ dos grupos 1 e 5 mostrou que os mesmos são constituídos, respectivamente, pelos sesquiterpenos conhecidos eudesma-5,7-dieno e eudesm6-en-4 $\beta$-ol. O grupo 2 foi submetido a uma coluna de gel de sílica impregnada com $\mathrm{AgNO}_{3}$ usando $\mathrm{CH}_{2} \mathrm{Cl}_{2}$ como fase móvel. Este procedimento gerou 45 frações que foram reunidas em 7 grupos após análise por CG. Destes, os grupos 6 e 7 estavam puros e compostos por $\beta$-selineno e por eudesma-4,11-dieno, os quais foram identificados por RMN de ${ }^{1} \mathrm{H} \mathrm{e} \mathrm{e}{ }^{13} \mathrm{C}$. Através da análise por CG, foi observado que os grupos 3, 6, e 9 são compostos por misturas que contêm respectivamente eudesma-5,7- 
dieno + $\beta$-selineno, guai-6-en-10 $\beta$-ol + eudesm-6-en$4 \beta$-ol e $5 \alpha, 6 \alpha, 7 \alpha, 8 \alpha$-diepoxi-eudesmano + eudesma5,7-dien-2 $\alpha$-ol. O grupo 7 foi submetido à cromatografia em camada delgada preparativa (gel de sílica) eluida com $\mathrm{CH}_{2} \mathrm{Cl}_{2}$ fornecendo guaia-6-en-4 $\beta$-ol na forma pura. O grupo 8 foi submetido também à cromatografia em camada delgada preparativa (gel de sílica) eluida com 1\% de $\mathrm{MeOH}$ em $\mathrm{CH}_{2} \mathrm{Cl}_{2}$ fornecendo três frações (8/1 - 8/3) compostas pelos sesquiterpenos $5 \alpha, 6 \alpha, 7 \alpha, 8 \alpha$-diepoxieudesmano, guai-6-en-10 $\beta$-ol e eudesma-5,7-dien-2 $\alpha$-ol, todos em suas formas puras. É digno de nota que esse último sesquiterpeno é um dos componentes minoritários do óleo bruto (1,6\%) e apresenta estrutura inédita na literatura, sendo possível sua caracterização somente após purificação e análise por RMN.

Todas os grupos provenientes das separações cromatográficas, tanto via $\mathrm{SiO}_{2}$ quanto por $\mathrm{SiO}_{2} /$ $\mathrm{AgNO}_{3}$, foram novamente avaliadas quanto ao potencial fungitóxico. A análise das placas aspergidas pelas soluções dos fungos indicou que o grupo 8 continha as substâncias ativas, visto que mostrou uma mancha branca sob fundo cinza, indicativo da inibição de crescimento dos mesmos (Homas; Fuchs, 1970). Um vez que esse grupo foi novamente fracionado, as frações 8/1 - 8/3 foram submetidas ao ensaio bioautográfico, mostrando que aquela composta pelo sesquiterpeno eudesma-5,7-dien$2 \alpha$-ol (8/3) era a responsável pelo potencial observado.

\section{CONCLUSÃO}

O isolamento e a purificação de compostos com estruturas raras e/ou desconhecidas, ou daqueles cuja análise por CG/EM e cálculo do índice de Kovats deixarem dúvidas quanto à identidade molecular, é uma necessidade inquestionável a fim determinar suas estruturas. Do mesmo modo, a obtenção de substâncias puras é essencial para que se possa reconhecer as moléculas responsáveis pelo potencial biológico apresentado por uma matriz complexa.

Desta forma, o procedimento proposto neste artigo constitui-se uma forma eficiente e fácil de ser aplicada em qualquer trabalho que vise à caracterização de componentes voláteis, principalmente para caracterização de moléculas de estruturas raras e/ou desconhecidas ou mesmo para purificação e conseqüente identificação de substâncias bioativas presentes em óleos voláteis.

\section{REFERÊNCIAS}

Adams RP 1995. Identification of Essential Oils by Ion Trap Mass Spectroscopy, Academic Press, San Diego.

Adio AM, Paul C, König WA, Muhle H 2002. Volatile components from European liverworts Marsupella emarginata, M. aquatica and M. alpina. Phytochemistry 61: 79-91.

Brochini CB, Núñez CV, Moreira IC, Chaves MH, Roque NF, Martins D 1999. Identificação de componentes de óleos voláteis: análise espectroscópica de misturas de sesquiterpenos. Quim Nova 22: 37-40.

Costantin MB, Sartorelli P, Ferreira MJP, Steppe M, Ohara M, Limberger R, Henriques AT, Emerenciano VP, Kato MJ 2001. Essential oils from Piper cernuum and Piper regnellii: Antimicrobial activities and analysis by GC/ MS and ${ }^{13} \mathrm{C}$ NMR. Planta Med 67: 771-773.

Ferreira MJP, Costantin MB, Sartorelli P, Rodrigues GV, Limberger R, Henriques AT, Kato MJ, Emerenciano VP 2001. Computer-aided method for identification of components in essential oils by 13C NMR spectroscopy. Anal Chim Acta 447: 125-134.

Gupta AS, Dev S 1963. Chromatography of organic compounds, I. Thin layer chromatography of olefins J Chromatogr 12: 189-190.

Homas AL, Fuchs A 1970. Direct bioautography in thin-layer chromatograms as a method for detecting fungitoxic substances. J Chromatogr 51: 327-329.

König WA, Bülow N, Fricke C, Melching S, Rieck A, Muhle H 1996. The sesquiterpene constituents of the liverwort Preissia quadrata. Phytochemistry 43: 629-633.

Lago JHG, Brochini CB, Roque NF 2000. Terpenes from leaves of Guarea macrophylla. Phytochemistry 55: 727-731.

Lago JHG, Roque NF 2002. Terpenes from essential oil from leaves of Guarea macrophylla ssp. tuberculata Vellozo (Meliaceae). J Essent Oil Res 14: 12-13.

Lago JHG, Brochini CB, Roque NF 2002. Terpenoids from Guarea guidonia. Phytochemistry 60: 333-338.

Lago JHG, Ramos CS, Casanova DCC, Morandin AA, Bergamo DCB, Cavalheiro AJ, Bolzani VS, Furlan M, Guimarães EF, Young MCM, Kato MJ 2004a. Benzoic acid derivatives from Piper species and their fungitoxic activity against Cladosporium cladosporioides and $C$. sphaerospermum. J Nat Prod 67: 1783-1788.

Lago JHG, Ávila Jr P, Aquino EM, Moreno PRH, Ohara MT, Limberger RP, Apel MA, Henriques AT 2004b. Volatile oils from leaves and stem barks of Cedrela fissilis (Meliaceae): chemical composition and antibacterial activities. Flavour Frag J 19: 448-451.

Mesquita JMO, Cavaleiro C, Cunha AP, Lombardi JA, Oliveira AB 2005. Estudo comparativo dos óleos voláteis de algumas espécies de Piperaceae. Rev Bras Farmacogn 15: 6-12.

Moreira IC, Roque NF, Contini K, Lago JHG 2007. Sesquiterpenos e hidrocarbonetos dos frutos de Xylopia emarginata (Annonaceae). Rev Bras Farmacogn 17: 55-58.

Nascimento PFC, Nascimento AC, Rodrigues CS, Antoniolli AR, Santos PO, Barbosa-Júnior AM, Trindade RC 2007. Atividade antimicrobiana dos óleos essenciais: uma abordagem multifatorial dos métodos. Rev Bras Farmacogn 17: 108-113.

Navickiene HMD, Morandim AA, Alécio AC, Regasini LO, Bergamo DCB, Telascrea M, Cavalheiro AJ, Lopes MN, Bolzani VS, Furlan M, Marques MOM, Young MCM, Kato MJ 2006. Composition and antifungal activity of essential oils from Piper aduncum, Piper arboreum and Piper tuberculatum. Quim Nova 26: 467-470.

Oliveira RN, Dias IJM, Câmara CAG 2005. Estudo comparativo do óleo essencial de Eugenia punicifolia (HBK) DC. de diferentes localidades de Pernambuco. Rev Bras Farmacogn 15: 39-42. 
Patitucci ML, Veiga Jr VF, Pinto AC, Zoghbi MGB, Silva JRA 1995. Utilização de cromatografia gasosa de alta resolução na detecção de classe de terpenos em extratos brutos vegetais. Quim Nova 18: 262-266.

Rahalison L, Hamburger M, Monod M, Frenk E, Hostettmann K 1994. Antifungal tests in phytochemical investigations - comparison of bioautographic methods using phytopathogenic and human phatogenic fungi. Planta Med 60: 41-44.

Souza AA, Laverde Jr A 2002. Aplicação de espectroscopia de ressonância magnética nuclear para estudos de difusão molecular em líquidos: a técnica DOSY. Quim Nova 25: 1022-1026.

Tavares ES, Julião LS, Lopes D, Bizzo HR, Lage CLS, Leitão SG 2005. Análise do óleo essencial de folhas de três quimiotipos de Lippia alba (Mill.) N. E. Br. (Verbenaceae) cultivados em condições semelhantes. Rev Bras Farmacogn 15: 1-5. 